\title{
Predicting oxygen vacancy non-stoichiometric concentration in perovskites from first principles
}

\author{
Heng Luo ${ }^{\mathrm{a}}$, Yongwoo Shin ${ }^{\mathrm{a}, \mathrm{b}}$, Yang Yu ${ }^{\mathrm{b}}$, Deniz Cetin ${ }^{\mathrm{b}}$, Karl Ludwig,c, \\ Uday Pal ${ }^{\mathrm{a}, \mathrm{b}}$, Soumendra N. Basu ${ }^{\mathrm{a}, \mathrm{b}}$, Srikanth Gopalan ${ }^{\mathrm{a}, \mathrm{b}}, \mathrm{Xi}$ Lin $^{\mathrm{a}, \mathrm{b}, *}$ \\ ${ }^{a}$ Department of Mechanical Engineering, Boston University, Boston, MA 02215, USA \\ ${ }^{b}$ Division of Materials Science and Engineering, Boston University, Brookline, MA \\ $02446, U S A$ \\ ${ }^{c}$ Department of Physics, Boston University, Boston, MA 02215, USA
}

\begin{abstract}
Formation of oxygen vacancies by introducing various mixed-valent cation dopants is a common practice to improve the cathode performance in solid oxide fuel cells. A computational procedure is developed in this work to predict the equilibrium oxygen vacancy non-stoichiometric concentrations at experimentally relevant temperatures and oxygen partial pressures for both bulk and surface oxide phases. The calculations are based on the first-principles density functional theory and a constrained free-energy functional. Quantitative agreements are found by direct comparisons to the thermogravimetry and solid electrolyte coulometry measurements for the strontium-doped lanthanum cobalt iron oxides at different compositions. Our results indicate that the oxygen vacancies are energetically stabilized at surfaces for all temperatures and all oxygen partial pressures, while such surface stabilization effects become stronger at higher temperatures and lower oxygen partial pressures.

Keywords: Solid oxide fuel cells, perovskite oxides, oxygen vacancy, non-stoichiometric concentration, first-principles density functional theory, free-energy functional
\end{abstract}

\footnotetext{
${ }^{*}$ Corresponding author

Email address: linx@bu.edu (Xi Lin)
} 


\section{Introduction}

Because of the unique combination of mixed ionic and electronic conductances (MIEC) [1, 2, 3, 4], perovskite oxides have been widely used as the cathode materials for solid oxide fuel cells (SOFC)[5]. Typical A-site cations in these perovskite cathode materials contain rare and alkaline earth elements such as $\mathrm{La}, \mathrm{Sr}, \mathrm{Ca}$, and $\mathrm{Ba}$, and typical B-site cations consist of reducible transition metal elements such as $\mathrm{Mn}, \mathrm{Fe}, \mathrm{Co}$, and $\mathrm{Ni}[6]$. Over the past two decades, extensive scientific research efforts have been focused on optimizing the materials compositions in lanthanum strontium transition metal oxides such as $\mathrm{La}_{1-x} \mathrm{Sr}_{x} \mathrm{Co}_{y} \mathrm{Fe}_{1-y} \mathrm{O}_{3-\delta}$ (LSCF) to improve the catalytic performance in oxygen reduction reactions, chemical and mechanical stabilities at high temperatures, and thermal expansion properties, as well as to lower the materials and processing costs $[7,8]$.

It has been well recognized that the MIEC of these perovskite cathode materials may be modified through controlling the oxygen non-stoichiometry $\mathrm{O}_{3-\delta}$ concentration $\delta[9]$, which in turn is a complicated function of the materials compositions, temperature $T$, and oxygen partial pressure $P_{\mathrm{O}_{2}}$. These non-stoichiometric anion vacancy defects were often introduced into perovskites in the growth, annealing, and redox reaction processes. However, if the resulting dopant and defect concentrations were not thermodynamically favorable, metastable microstructures could undergo degradations[10] and consequentially affect the overall device performance.

Experimentally, oxygen vacancy non-stoichiometric concentrations in LSCF were measured through oxygen mass changes using the thermo-gravimetry (TG)[9] and solid electrolyte coulometry (SECM)[11] techniques. Various empirical defect models $[9,12,13]$ were introduced to explain these measurements, but all shared the following common problems. Firstly, all the cation and anion defects were treated as classical particles existing only in their pre-assigned integer valence states and only on the lattice sites such that the quantum nature of electrons and holes was completely ignored. Secondly, these defect models typically required three or four empirical fitting parameters in the forms of enthalpy, entropy, or equilibrium constants. But these fitted thermodynamical functions did not have any explicit temperature and pressure dependences. Thirdly, although the importance of surface defects were often acknowledged in the perovskite oxide literatures $[9,13]$, these existing empirical defect models $[9,12,13]$ did not consider any surface effects. In this work, we present a first-principles density functional theory (DFT) 
based free-energy approach to predict the oxygen vacancy non-stoichiometric concentrations $\mathrm{O}_{3-\delta}$ for the well documented LSCF system. In this new approach, the cation and anion defects are allowed to displace away from welldefined lattice sites, and their valence states are the natural consequence of continuous wave function and charge distributions. With only one single excess Gibbs free-energy parameter describing the non-ideal solution effects which can be estimated from existing experimental measurements, we expect that the same numerical procedures descried in this work for LSCF can also be applicable to other perovskite oxide materials. To the best of our knowledge, our present approach is the only model that explicitly incorporate the essential surface effects on the oxygen vacancy non-stoichiometric concentrations.

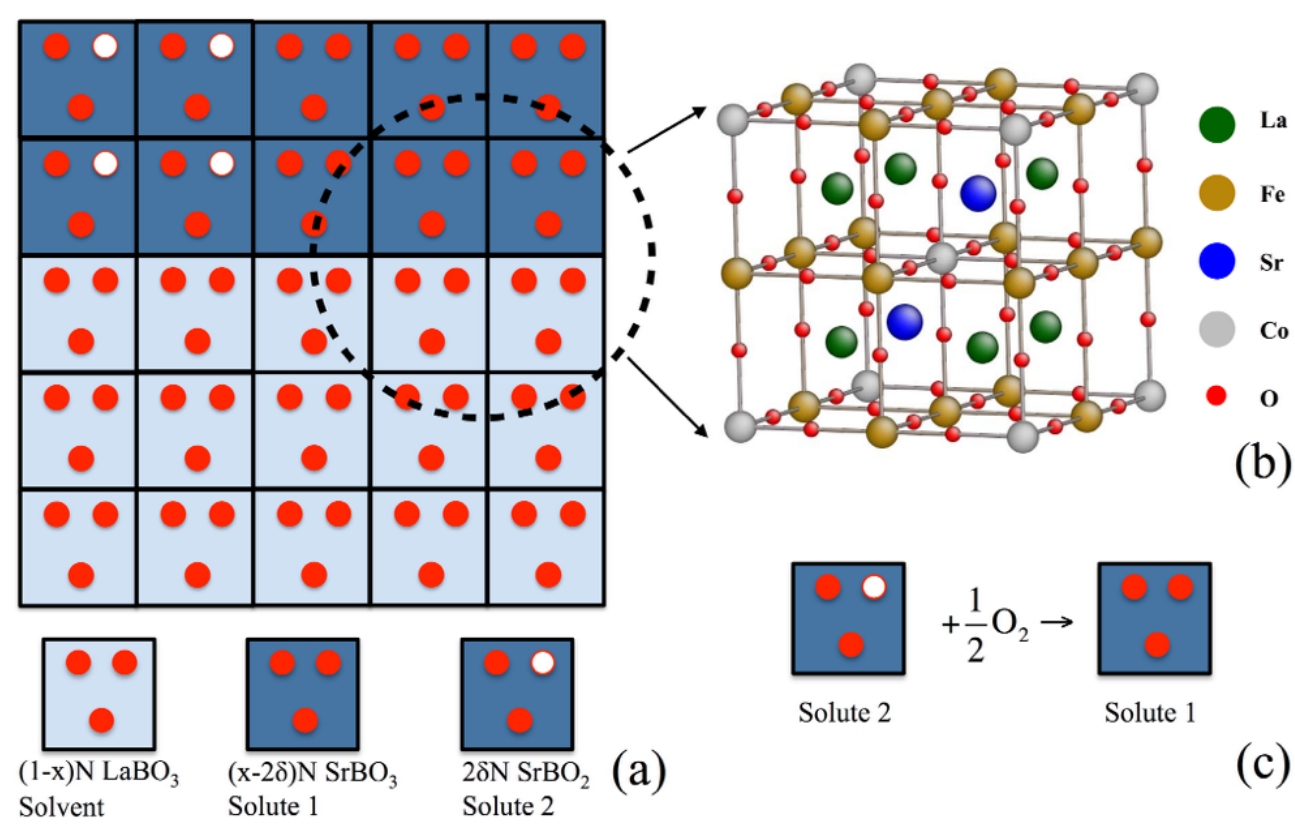

Figure 1: (a) Schematic supercell ensemble, where the dark and light blue cells represent the Sr and La unit cells, respectively. A red filled circle indicates that the corresponding oxygen site is filled with an oxygen atom, and an empty red circle represents an oxygen vacancy site. The chance of forming two oxygen vacancies in one single unit cell is ignored. (b) Representative $2 \times 2 \times 2$ supercell of $\mathrm{La}_{0.75} \mathrm{Sr}_{0.25} \mathrm{Co}_{0.25} \mathrm{Fe}_{0.75} \mathrm{O}_{3}$ used in our DFT calculations. (c) Because the oxygen vacancy formation energy in a La unit cell is much greater $(>1.2 \mathrm{eV})$ than that in a $\mathrm{Sr}$ unit cell, the $\mathrm{LaBO}_{3}, \mathrm{SrBO}_{3}$, and $\mathrm{SrBO}_{2}$ cells are treated in our free-energy model as the Solvent, Solute 1, and Solute 2, respectively. 


\section{Computational details}

Electronic-structure calculations based on the first-principles DFT provides a generic and powerful tool for predicting many important thermodynamic and kinetic properties of complex oxides[14, 15, 16, 17]. The ground state energy calculations are performed using the standard Quantum Espresso software package[18], which gives all the equilibrium crystal structures, atomic geometries, vibrational entropies under the harmonic oscillator approximation, and electronic wave function and density distributions.

We used plane waves as the basis set with an energy cutoff of $450 \mathrm{eV}$, ultrasoft pseudopotentials, and generalized gradient approximation (GGA) for the exchange-correlation functional with the on-site Hubbard repulsion $\mathrm{U}(\mathrm{GGA}+\mathrm{U})[19]$ corrections. Standard Coulomb repulsion parameter $U$ $=5 \mathrm{eV}$ and exchange parameter $J=1 \mathrm{eV}$ are applied to the $3 d$ orbitals of the B-site transition metals including Fe and $\mathrm{Co}[14]$. The spin polarization effects are fully treated in all the calculations. A representative supercell with the periodic boundary conditions applied along all the three $x, y$, and $z$ directions is given in Fig. 1(b) for $\mathrm{La}_{0.75} \mathrm{Sr}_{0.25} \mathrm{Co}_{0.25} \mathrm{Fe}_{0.75} \mathrm{O}_{3}$ which contain a total of 40 atoms. A corresponding $2 \times 2 \times 2$ Monkhorst-Pack $k$-point mesh in the first Brillouin zone was used for the total energy calculations[20]. The equilibrium lattice constant of such a cubic perovskite oxide is $3.91 \AA$ computed by DFT, consistent with the experimental value of $3.925 \AA$ for $\mathrm{La}_{0.6} \mathrm{Sr}_{0.4} \mathrm{Co}_{0.2} \mathrm{Fe}_{0.8} \mathrm{O}_{3}[21]$. To mimic the surface phase, a large vacuum of 10 $\AA$ is used and the atoms on the bottom layer are fixed at their bulk geometry.

Going beyond the total energy calculations directly from DFT, we need to construct a free-energy functional to obtain the oxygen vacancy nonstoichiometry concentration as a function of $T$ and $P_{\mathrm{O}_{2}}$. As shown in Fig. 1(a), a macroscopic sample LSCF perovskite oxide may be represented as an ensemble of $N$ cubic perovskite unit cells. In each unit cell, there are one A atomic site, one B atomic site, and three oxygen atomic sites. For a LSCF sample with a Sr molar dopant fraction of $x$, there are $x N$ Sr-containing perovskite unit cells and $(1-x) N$ La-containing perovskite unit cells, corresponding to the dark blue and light blue unit cells shown in Fig. 1(a) respectively.

Because it is highly energetically unfavorable to create oxygen vacancies in the La-containing cells as compared to the Sr-containing cells and it is both energetically and entropically unfavorable to create two oxygen vacancies

in one single Sr-containing cell, we can further divide the LSCF supercell 
ensemble of Fig. 1(a) into three different categories, $(1-x) N \mathrm{LaBO}_{3}$ as the Solvent, $(x-2 \delta) N \mathrm{SrBO}_{3}$ as the Solute 1, and $2 \delta N \mathrm{SrBO}_{2}$ as the Solute 2. Therefore, the oxygen vacancy formation can only occur between the Solute 1 and Solute 2, as shown in Fig. 1(c). Here the charge neutrality condition is enforced between the $\mathrm{Sr}$ dopants and $\mathrm{O}$ vacancies as

$$
\left[\mathrm{Sr}_{\mathrm{La}}^{\prime}\right]=2\left[\mathrm{~V}_{\mathrm{O}}^{\bullet \bullet}\right]
$$

Following such an LSCF defect ensemble representation as shown in Fig. 1, we can express the overall reaction Gibbs free-energy

$$
\Delta G=\Delta H-T \Delta S
$$

as the sum of the reaction enthalpy

$$
\Delta H=H_{\text {Solute } 1}-H_{\text {Solute } 2}-\frac{1}{2} H_{\mathrm{O}_{2}},
$$

directly from DFT and the reaction entropy

$$
\Delta S=\Delta S_{\text {solid }}-\frac{1}{2} \Delta S_{\text {gas }}=\left(\Delta S_{\text {config }}+\Delta S_{\text {vib }}\right)-\frac{1}{2} \Delta S_{\mathrm{O}_{2}} .
$$

As shown in Eq. (4), the latter consists of the configurational entropy and vibrational entropy contributions of the solids, as well as the entropy contribution of the gas-phase molecules. Assuming the ideal mixing of all the different supercells in Fig. 1(a), we have

$$
\Delta S_{\text {config }}=-k_{\mathrm{B}}[2 \delta \ln (2 \delta)+(x-2 \delta) \ln (x-2 \delta)] .
$$

Non-ideal free-energy contributions to both the enthalpy term of Eq. (3) and the configurational entropy term of Eq. (5) can be obtained by comparing to real experimental measurements, as to be discussed below.

At experimentally relevant temperatures and pressures, since the vibrational entropy contribution $\Delta S_{\text {vib }}$ to both the thermodynamic $[22,23,24]$ and kinetic[25] properties in typical heterogeneous chemical reactions are much smaller than the other two entropy terms, $\Delta S_{\text {vib }}$ will be neglected in this work for simplicity. The gas-phase $\Delta S_{\mathrm{O}_{2}}$ can be either computed from DFT via the canonical transformation of phonons fed to the standard ideal gas, rigid rotor, and harmonic oscillator partition functions[24] or simply adapted from the NIST Webbook[26] as in this work. Their differences in $\Delta S_{\mathrm{O}_{2}}$ at 
experimentally relevant temperatures are typically negligible[24]. Finally, by combining Eqs. (2)-(5), we find an expression for $\Delta G$ as

$$
\Delta G=\Delta H-T \Delta S_{\text {config }}+\frac{1}{2} T \Delta S_{\mathrm{O}_{2}} .
$$

When the chemical reaction of Fig. 1(c) reaches thermal equilibrium, the reaction equilibrium constant $K_{\text {eq }}$ becomes

$$
K_{\mathrm{eq}}=\frac{a_{\text {Solute } 1}}{a_{\text {Solute } 2} a_{\mathrm{O}_{2}}{ }^{\frac{1}{2}}}=\exp \left[-\frac{\Delta G}{\mathrm{RT}}\right],
$$

where $R$ is the universal gas constant and the corresponding activities can be written as

$$
\begin{gathered}
a_{\text {Solute1 }}=\gamma_{\text {Solute1 }} \times(x-2 \delta), \\
a_{\text {Solute2 }}=\gamma_{\text {Solute } 2} \times(2 \delta),
\end{gathered}
$$

and

$$
a_{\mathrm{O}_{2}}=\frac{P_{\mathrm{O}_{2}}}{p^{0}}
$$

where $p^{0}$ is the standard 1 atmosphere (atm) reference state.

Defining the activity coefficient ratio $\gamma_{\mathrm{r}}=\gamma_{\text {Solute1 }} / \gamma_{\text {Solute2 }}$, we can rewrite the equilibrium constant equation as

$$
\begin{aligned}
\gamma_{\mathrm{r}} \frac{x-2 \delta}{2 \delta} & =\exp \left[-\frac{\Delta G}{\mathrm{R} T}\right]\left[\frac{P_{\mathrm{O}_{2}}}{p_{0}}\right]^{\frac{1}{2}} \\
& =\exp \left[-\frac{\Delta H}{\mathrm{R} T}+\frac{\Delta S_{\text {config }}}{\mathrm{R}}-\frac{\Delta S_{\mathrm{O}_{2}}}{2 \mathrm{R}}\right]\left[\frac{P_{\mathrm{O}_{2}}}{p_{0}}\right]^{\frac{1}{2}} .
\end{aligned}
$$

Here the complicated activity coefficient ratio, which is related to the excess Gibbs energy beyond the ideal solution model, will be estimated from the experimentally measured $\delta$ data as to be discussed below.

\section{Results and discussion}

The thermodynamic energetics computed by DFT were given as follows

$$
\begin{aligned}
H(\delta) & =H_{\mathrm{La}_{1-x} \mathrm{Sr}_{x} \mathrm{Co}_{y} \mathrm{Fe}_{1-y} \mathrm{O}_{3-\delta}}+\frac{\delta}{2} H_{\mathrm{O}_{2}} \\
& -H_{\mathrm{La}_{1-x} \mathrm{Sr}_{x} \mathrm{Co}_{y} \mathrm{Fe}_{1-y} \mathrm{O}_{3}}(\mathrm{Bulk}),
\end{aligned}
$$


where the perfect bulk phase without vacancies was chosen to be the energy reference. Fig. 2 shows the DFT vacancy formation energy for one particular LSCF composition $\mathrm{La}_{0.75} \mathrm{Sr}_{0.25} \mathrm{Co}_{0.25} \mathrm{Fe}_{0.75} \mathrm{O}_{3-\delta}$. It is clear that the formation of surface vacancy is much lower than that in the bulk phase. Such a direct correlation between the vacancy formation and the free surface is consistent with the experimental observed segregations near the LSCF surfaces[27].

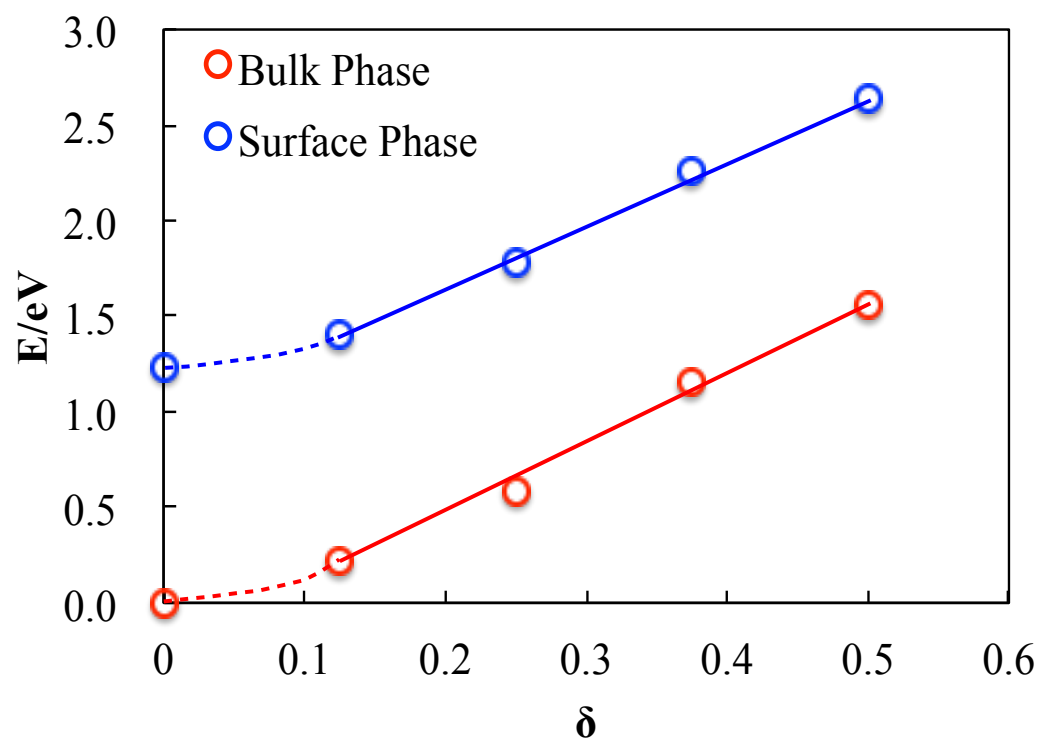

Figure 2: Vacancy formation energy of $\mathrm{La}_{0.75} \mathrm{Sr}_{0.25} \mathrm{Co}_{0.25} \mathrm{Fe}_{0.75} \mathrm{O}_{3-\delta}$ as a function of the vacancy concentration $\delta$ is computed by DFT for both the bulk (red) and surface (blue) phases. The perfect bulk phase is used as the common energy reference.

Inserting the DFT enthalpy results of Fig. 2 into Eq. (11), we can iteratively solve for the oxygen vacancy non-stoichiometric concentrations as a function of $P_{\mathrm{O}_{2}}$ for both the bulk and surface phases, plotted in Fig. 3 as the solid and dashed lines respectively. Together shown in Fig. 3 are the experimental TG (red circles)[9] and SECM (blue crosses)[11] data at four different temperatures $T=873 \mathrm{~K}, 973 \mathrm{~K}, 1073 \mathrm{~K}$, and $1173 \mathrm{~K}$. It is clear that the experimental data are located inside of the shaded area between the bulk phase limit and the surface phase limit, which indicates that the experimental data is a combination of the bulk and surface phases and the two samples from two different groups are likely to have slightly different 

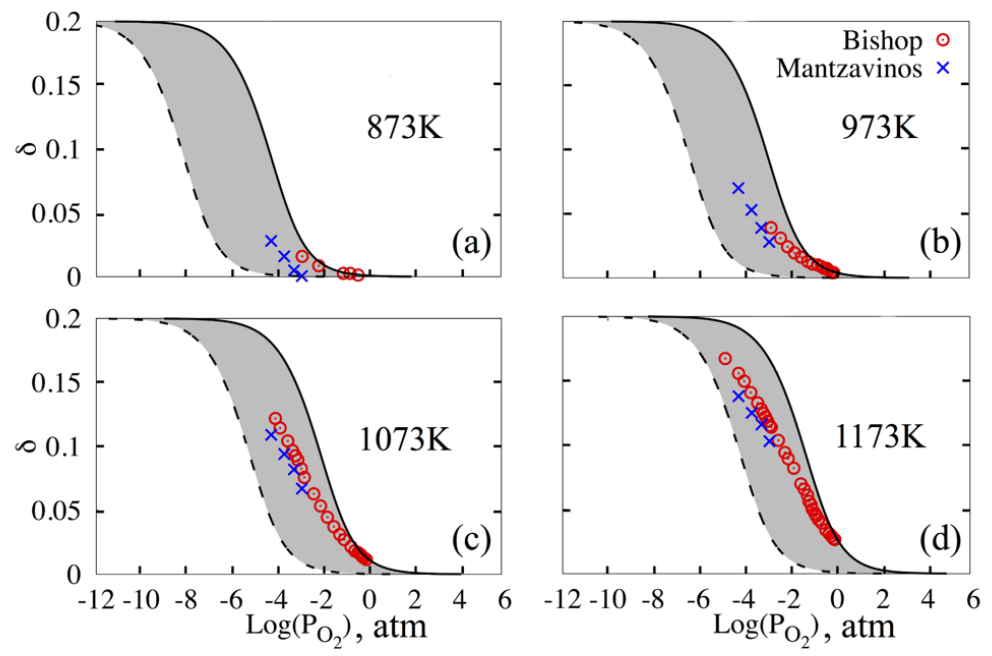

Figure 3: Oxygen vacancy concentration $\delta$ as a function of oxygen partial pressure $P_{\mathrm{O}_{2}}$ at four different temperatures $T=873,973,1073$, and $1173 \mathrm{~K}$. The dashed and solid lines represent the calculated bulk and surface phase limits by DFT, respectively. The experimental data, TG (red circles) by Bishop et al.[9] and SECM (blue crosses) by Mantzavinos et al.[11], are located in the shaded area between the computed surface and bulk phase limits. As the vacancy concentration increases, the experimental data gradually approach from the surface limit to the bulk limit. Note that due to the computational supercell limitation, LCSF with a slightly different composition is used in the DFT calculations $\mathrm{La}_{0.75} \mathrm{Sr}_{0.25} \mathrm{Co}_{0.25} \mathrm{Fe}_{0.75} \mathrm{O}_{3-\delta}$ as compared to the experimental sample of $\mathrm{La}_{0.6} \mathrm{Sr}_{0.4} \mathrm{Co}_{0.2} \mathrm{Fe}_{0.8} \mathrm{O}_{3-\delta}$ (LSCF 6428).

bulk-to-surface ratios. It is important to note that since the vacancy formation energy at surfaces are much less than that in the bulk phase (Fig. 2). Therefore, multiple-parameter fitting with no explicit surface dependences in all existing empirical models may not necessarily have any physical meanings. The essential role of surfaces on many thermodynamic properties of these perovskite oxides has been well acknowledged in literatures[9].

At any fixed temperature among all the four temperatures shown in Fig. 3 , as $P_{\mathrm{O}_{2}}$ decreases the oxygen vacancy concentration $\delta$ not only increases as expected, but also gradually switches from the bulk phase limit towards the surface phase limit. This new feature was missing in all the previous empirical models [9]. Physically, this trend implies that when $P_{\mathrm{O}_{2}}$ is high, there are only a small quantity of vacancies in the entire system, and since vacancies prefer the surface face (Fig. 2) they would prefer to be close to the surface phase. 
On the other hand, when the $P_{\mathrm{O}_{2}}$ is low, more and more imperfect unit cells appear in the system. Because the number of the imperfect unit cells in the surface phase is limited, more and more imperfect unit cells will be located toward the bulk phase such that the experimentally measured oxygen vacancy concentration $\delta$ switches toward the bulk phase limit as shown in Fig. 3. Overall, the DFT results show good agreement with the experimental data within one single unknown physical parameter, the activity coefficient ratio $\gamma_{\mathrm{r}}$ of Eq. (11), which indicates that the calculated DFT energetics and the estimated reaction model are capable of describing the complicated defect chemistry of LSCF.

Additionally, we can reformulate such a complicated activity coefficient ratio of Eq. (11) into the excess Gibbs free energy formalism due to the non-ideal mixing effects ignored in Fig. 1. Namely,

$$
\gamma_{\mathrm{r}}=\exp \left[\frac{\Delta G_{\text {non-ideal }}}{\mathrm{R} T}\right]=\exp \left[\frac{\Delta H_{\text {non-ideal }}}{\mathrm{R} T}\right] \exp \left[\frac{\Delta S_{\text {non-ideal }}}{\mathrm{R}}\right]
$$

Indeed, plotting $\log \left(\gamma_{\mathrm{r}}\right)$ versus $1 / T$ in Fig. 4, we find such a linear relationship is confirmed.

Therefore, combining Fig. 3, Fig. 4 and Eq. (11), it is clear that the LSCF data from both the TG and SECM measurements are a combination of vacancies from both the bulk phase and the surface phase. Moreover, we can estimate the surface phase fraction of the overall vacancy concentration by defining

$$
\text { Surface Phase Fraction }=\frac{\delta_{\text {Experiment }}-\delta_{\mathrm{B}}}{\delta_{\mathrm{S}}-\delta_{\mathrm{B}}} .
$$

The corresponding results as a function of $P_{\mathrm{O}_{2}}$ are shown in Fig. 5 for two representative temperatures. For both temperatures, the surface phase fraction decreases when the oxygen partial pressure $P_{\mathrm{O}_{2}}$ decreases. Such a surface-tobulk transition occurs at different $P_{\mathrm{O}_{2}}$ windows for different temperature. In general, it occurs at lower partial pressure values if the temperature is lower as shown in Fig. 5.

Combining the reformulated $T$-dependent activity coefficient ratio obtained from Fig. 4 and Eq. (11), we can now predict the oxygen vacancy non-stoichiometric concentration $\delta$ as a continuous function of $T$ and $P_{\mathrm{O}_{2}}$. The constant $\delta$ contour plots are shown in Fig. 6, where the red, green, and blue lines represent $\delta=0.15,0.10$, and 0.05 , respectively. Only three iso-value lines of the oxygen vacancy concentration are shown for both the 


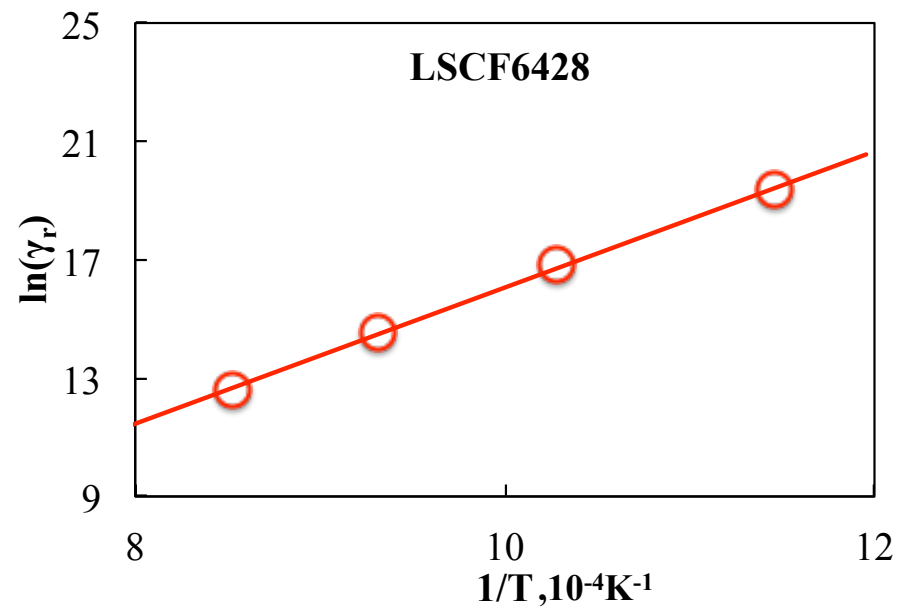

Figure 4: The unknown activity coefficient ratio $\gamma_{\mathrm{r}}$ is reformulated into the Gibbs free energy changes due to non-ideal mixing, as confirmed by the linear relationship between $\log \left(\gamma_{\mathrm{r}}\right)$ and $1 / T$.

surface and bulk phases to avoid ambiguous overlaps. Using the predicted $\delta_{\mathrm{B}}$ and $\delta_{\mathrm{S}}$ contours as the guidelines, we can give a reasonable estimation of the equilibrium $\delta$ for real experimental samples.

Using the same computational procedure discussed above, we can also compute the oxygen nonstoichiometric concentrations for LSCF with different materials compositions. Explicitly, Fig. 7 shows the direct comparison of our computed $\mathrm{La}_{0.8} \mathrm{Sr}_{0.2} \mathrm{Co}_{0.2} \mathrm{Fe}_{0.8} \mathrm{O}_{3-\delta}$ (LSCF8228) bulk and surface limits with the SECM data measured by Mantzavinos et al.[11].

\section{Summary and conclusions}

A first-principles DFT based computational approach is developed in this work to predict the oxygen vacancy non-stoichiometric concentrations for LSCF as a function of temperature and partial pressure of oxygen. Good agreements with the experimental data are found over the entire relevant temperature and pressure ranges. Our prediction indicates that the oxygen vacancy non-stoichiometric concentrations in real materials samples have contributions from both the surface phase and the bulk phase. The surface contributions are stronger at lower temperatures and at higher oxygen partial pressures. The fast surface-to-bulk phase transition occurs at lower partial 


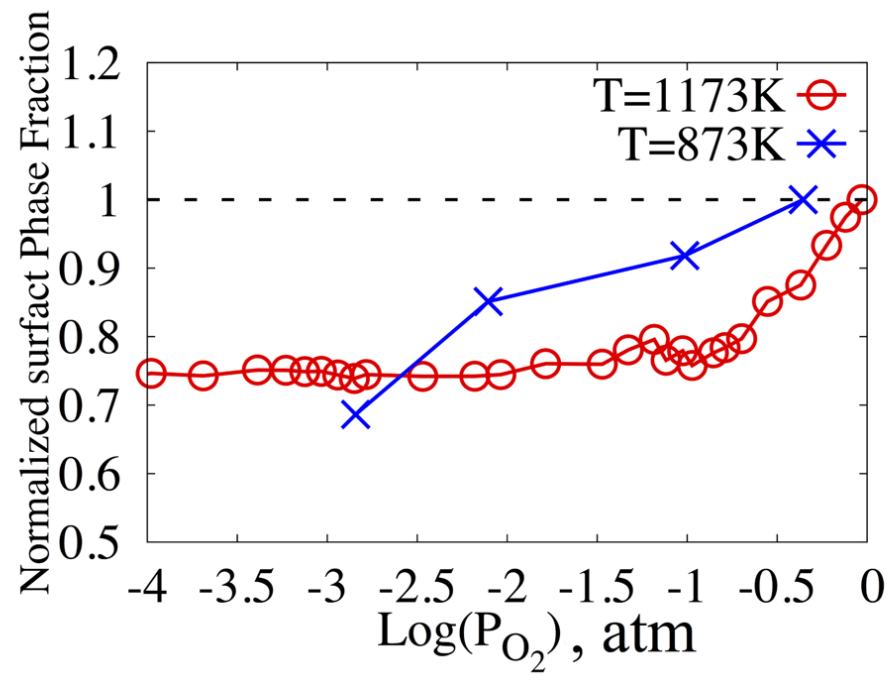

Figure 5: Normalized surface phase fraction versus the oxygen partial pressure $P_{\mathrm{O}_{2}}$ for two representative temperatures. For both temperatures, the surface phase fraction decreases as $P_{\mathrm{O}_{2}}$ decreases. This fast bulk-to-surface crossover occurs at lower partial pressures for lower temperatures.

pressure windows for lower temperatures.

The work is supported by DOE-SECA under Grant DEFC2612FE0009656 and NSF-XSEDE under Grant DMR-0900073.

[1] H. Anderson, Review of p-type doped perovskite materials for SOFC and other applications, Solid State Ionics 52 (1992) 33-41.

[2] P. Dyer, R. Richards, S. Russek, D. Taylor, Ion transport membrane technology for oxygen separation and syngas production, Solid State Ionics 134 (2000) 21-33.

[3] H. Wang, Y. Cong, W. Yang, Investigation on the partial oxidation of methane to syngas in a tubular $\mathrm{Ba}_{0.5} \mathrm{Sr}_{0.5} \mathrm{Co}_{0.8} \mathrm{Fe}_{0.2} \mathrm{O}_{3-\delta}$ membrane reactor, Catalysis Today 82 (1-4) (2003) 157-166.

[4] Y. Liu, X. Tan, K. Li, Mixed Conducting Ceramics for Catalytic Membrane Processing, Catalysis Reviews 48 (2) (2006) 145-198.

[5] C. Sun, R. Hui, J. Roller, Cathode materials for solid oxide fuel cells: a review, Journal of Solid State Electrochemistry 14 (7) (2009) 1125-1144. 


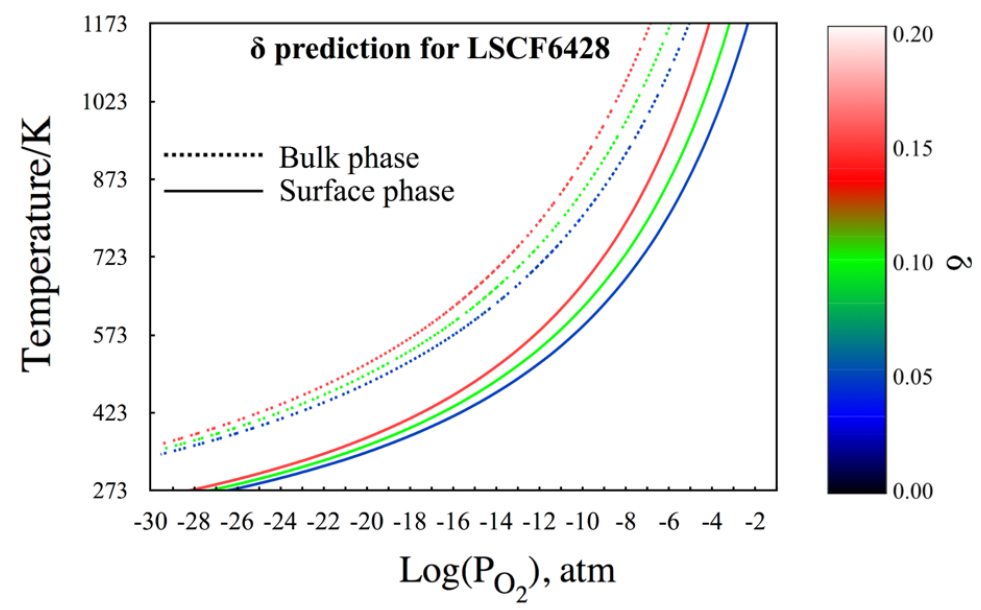

Figure 6: Predicted oxygen vacancy non-stoichiometric concentration $\delta$ for LSCF6428 as a function of $P_{\mathrm{O}_{2}}$ and $T$ for both the bulk (solid lines) and surface (dashed) phases. Only three iso-value lines for three different oxygen vacancy concentrations are explicitly shown, red for $\delta=0.15$, green for $\delta=0.10$, and blue for $\delta=0.05$. For experimental samples, the oxygen vacancy non-stoichiometric concentrations $\delta$ are between the bulk and surfaced limits.

[6] M. A. Pena, J. L. G. Fierro, Chemical Structures and Performance of Perovskite Oxides, Chemical Reviews 101 (2001) 1981-2017.

[7] B. C. H. Steele, Survey of materials selection for ceramic fuel cells, Solid State Ionics 88 (1996) 1223-1234.

[8] V. Dusastre, J. Kilner, Optimisation of composite cathodes for intermediate temperature SOFC applications, Solid State Ionics 126 (1999) $163-174$.

[9] S. R. Bishop, K. L. Duncan, E. D. Wachsman, Surface and bulk defect equilibria in strontium-doped lanthanum cobalt iron oxide, Journal of The Electrochemical Society 156 (10) (2009) B1242-B1248.

[10] G. Y. Yang, E. C. Dickey, C. A. Randall, M. S. Randall, L. A. Mann, Modulated and ordered defect structures in electrically degraded $\mathrm{Ni}-\mathrm{BaTiO}_{3}$ multilayer ceramic capacitors, Journal of Applied Physics 94 (9) (2003) 5990. 

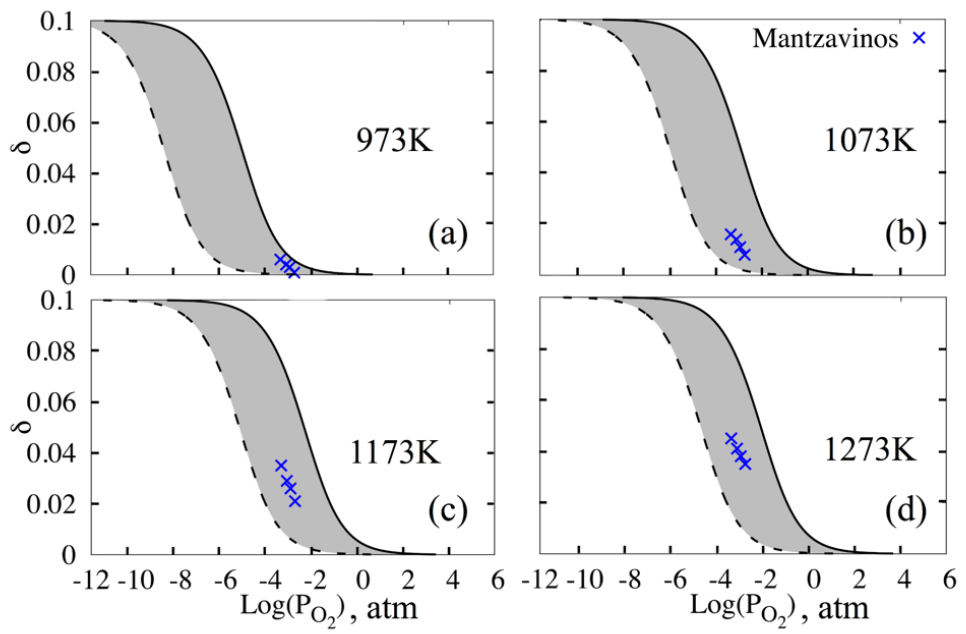

Figure 7: Oxygen vacancy concentration $\delta$ as a function of oxygen partial pressure $P_{\mathrm{O}_{2}}$ for LSCF8228. The dashed and solid lines represent the calculated bulk phase and surface phase limits. The experimental data measured by Mantzavinos[11] using the SECM technique are located inside of the shaded area, indicating the real materials samples are combination of these two phases.

[11] D. Mantzavinos, A. Hartley, I. S. Metcalfe, M. Sahibzada, Oxygen stoichiometries in $\mathrm{La}_{1-\mathrm{x}} \mathrm{Sr}_{\mathrm{x}} \mathrm{Co}_{1-\mathrm{y}} \mathrm{Fe}_{\mathrm{y}} \mathrm{O}_{3-\delta}$ perovskites at reduced oxygen partial pressures, Solid State Ionics 134 (2000) 103-109.

[12] S. Stø len, E. Bakken, C. E. Mohn, Oxygen-deficient perovskites: linking structure, energetics and ion transport., Physical chemistry chemical physics 8 (4) (2006) 429-47.

[13] E. Bakken, T. Norby, S. Stø len, Redox energetics of perovskite-related oxides, Journal of Materials Chemistry 12 (2) (2002) 317-323.

[14] Y.-L. Lee, J. Kleis, J. Rossmeisl, D. Morgan, Ab initio energetics of $\mathrm{LaBO}_{3}(001)$ ( $\mathrm{B}=\mathrm{Mn}, \mathrm{Fe}, \mathrm{Co}$, and $\mathrm{Ni}$ ) for solid oxide fuel cell cathodes, Physical Review B 80 (22) (2009) 224101.

[15] M. J. Gadre, Y.-L. Lee, D. Morgan, Cation interdiffusion model for enhanced oxygen kinetics at oxide heterostructure interfaces., Physical chemistry chemical physics 14 (8) (2012) 2606-16. 
[16] Y. A. Mastrikov, R. Merkle, E. A. Kotomin, M. M. Kuklja, J. Maier, Formation and migration of oxygen vacancies in $\mathrm{La}_{1-\mathrm{x}} \mathrm{Sr}_{\mathrm{x}} \mathrm{Co}_{1-\mathrm{y}} \mathrm{Fe}_{\mathrm{y}} \mathrm{O}_{3-\delta}$ perovskites: insight from ab initio calculations and comparison with $\mathrm{Ba}_{1-x} \mathrm{Sr}_{\mathrm{x}} \mathrm{Co}_{1-y} \mathrm{Fe}_{\mathrm{y}} \mathrm{O}_{3-\delta}$., Physical chemistry chemical physics 15 (3) (2013) 911-8.

[17] R. Merkle, Y. A. Mastrikov, E. A. Kotomin, M. M. Kuklja, J. Maier, First Principles Calculations of Oxygen Vacancy Formation and Migration in $\mathrm{Ba}_{1-\mathrm{x}} \mathrm{Sr}_{\mathrm{x}} \mathrm{Co}_{1-\mathrm{y}} \mathrm{Fe}_{\mathrm{y}} \mathrm{O}_{3-\delta}$ Perovskites, Journal of The Electrochemical Society 159 (2) (2012) B219.

[18] P. Giannozzi, S. Baroni, N. Bonini, M. Calandra, R. Car, C. Cavazzoni, D. Ceresoli, G. L. Chiarotti, M. Cococcioni, I. Dabo, A. Dal Corso, S. de Gironcoli, S. Fabris, G. Fratesi, R. Gebauer, U. Gerstmann, C. Gougoussis, A. Kokalj, M. Lazzeri, L. Martin-Samos, N. Marzari, F. Mauri, R. Mazzarello, S. Paolini, A. Pasquarello, L. Paulatto, C. Sbraccia, S. Scandolo, G. Sclauzero, A. P. Seitsonen, A. Smogunov, P. Umari, R. M. Wentzcovitch, QUANTUM ESPRESSO: a modular and open-source software project for quantum simulations of materials., Journal of physics. Condensed matter 21 (39) (2009) 395502.

[19] A. I. Liechtenstein, V. I. Anisimov, J. Zaanen, Density-functional theory and strong interactions: Orbital ordering in mott-hubbard insulators, Phys. Rev. B 52 (1995) R5467-R5470.

[20] H. J. Monkhorst, J. D. Pack, Special points for brillouin-zone integrations, Phys. Rev. B 13 (1976) 5188-5192.

[21] J. S. Hardy, J. W. Templeton, D. J. Edwards, Z. Lu, J. W. Stevenson, Lattice expansion of LSCF-6428 cathodes measured by in situ XRD during SOFC operation, Journal of Power Sources 198 (2012) 76-82.

[22] X. Lin, N. J. Ramer, A. M. Rappe, K. C. Hass, W. F. Schneider, B. L. Trout, Effect of particle size on the adsorption of o and s atoms on pt: A density-functional theory study, Journal of Physical Chemistry B 105 (2001) 7739-7747.

[23] X. Lin, K. C. Hass, W. F. Schneider, B. L. Trout, Chemistry of sulfur oxides on transition metals i. configurations, energetics, orbital analyses, and surface coverage effects of $\mathrm{so}_{2}$ on $\mathrm{pt}(111)$, Journal of Physical Chemistry B 106 (2002) 12575-12583. 
[24] X. Lin, W. F. Schneider, B. L. Trout, Chemistry of sulfur oxides on transition metals. ii. thermodynamics of sulfur oxides on platinum(111), Journal of Physical Chemistry B 108 (2004) 250-264.

[25] X. Lin, W. F. Schneider, B. L. Trout, Chemistry of sulfur oxides on transition metals. iii. oxidation of $\mathrm{so}_{2}$ and self-diffusion of $\mathrm{o}_{2} \mathrm{SO}_{2}$, and $\mathrm{so}_{3}$ on pt(111), Journal of Physical Chemistry B 108 (2004) 13329-13340.

[26] http://webbook.nist.gov/.

[27] S. P. Simner, M. D. Anderson, M. H. Engelhard, J. W. Stevenson, Degradation Mechanisms of $\mathrm{La}-\mathrm{Sr}-\mathrm{Co}-\mathrm{Fe}-\mathrm{O}_{3}$ SOFC Cathodes, Electrochemical and Solid-State Letters 9 (10) (2006) A478. 